\title{
Toward Value Based Health Care in pituitary surgery: application of a comprehensive outcome set in perioperative care
}

\author{
Daniel J Lobatto ${ }^{1,2}$, Amir H Zamanipoor Najafabadi ${ }^{1,2}$, Friso de Vries ${ }^{1,3}$, Cornelie D Andela ${ }^{1,3}$, \\ Wilbert B van den Hout ${ }^{1,4}$, Alberto M Pereira ${ }^{1,3}$, Wilco C Peul ${ }^{1,2,4,5}$, Thea P M Vliet Vlieland ${ }^{1,6}$, \\ Wouter $R$ van Furth ${ }^{1,2}$ and Nienke R Biermasz ${ }^{1,3}$
}

${ }^{1}$ Center for Endocrine Tumors Leiden, ${ }^{2}$ Department of Neurosurgery, ${ }^{3}$ Division of Endocrinology, Department of Medicine, ${ }^{4}$ Medical Decision Making, Department of Biomedical Data Sciences, Leiden University Medical Center, Leiden, The Netherlands, ${ }^{5}$ Department of Neurosurgery, Haaglanden Medical Center, The Hague, The Netherlands, and ${ }^{6}$ Department of Orthopedics, Physical Therapy and Rehabilitation, Leiden University Medical Center, Leiden, The Netherlands

Correspondence should be addressed to D J Lobatto

Email

d.j.lobatto@lumc.nl

\begin{abstract}
Objective: Although widely advocated, applying Value Based Health Care (VBHC) in clinical practice is challenging. This study describes VBHC-based perioperative outcomes for patients with pituitary tumors up to 6 months postoperatively.

Methods: A total of 103 adult patients undergoing surgery were prospectively followed. Outcomes categorized according to the framework of VHBC included survival, degree of resection, endocrine remission, visual outcome (including self-perceived functioning), recovery of pituitary function, disease burden and health-related quality of life (HRQoL) at 6 months (Tier 1); time to recovery of disease burden, HRQoL, visual function (Tier 2); permanent hypopituitarism and accompanying hormone replacement (Tier 3). Generalized estimating equations (GEEs) analysis was performed to describe outcomes over time.

Results: Regarding Tier 1, there was no mortality, 72 patients (70\%) had a complete resection, 31 of 45 patients (69\%) with functioning tumors were in remission, 7 (12\%, with preoperative deficits) had recovery of pituitary function and 45 of 47 (96\%) had visual improvement. Disease burden and HRQoL improved in 36-45\% at 6 months; however, there were significant differences between tumor types. Regarding Tier 2: disease burden, HRQoL and visual functioning improved within 6 weeks after surgery; however, recovery varied widely among tumor types (fastest in prolactinoma and non-functioning adenoma patients). Regarding Tier 3, 52 patients (50\%) had persisting (tumor and treatmentinduced) hypopituitarism.

Conclusions: Though challenging, outcomes of a surgical intervention for patients with pituitary tumors can be reflected through a VBHC-based comprehensive outcome set that can distinguish outcomes among different patient groups with respect to tumor type.
\end{abstract}

\section{Introduction}

Pituitary tumors are rare tumors of endocrine origin (1, 2 ), which can cause systemic signs and symptoms due to hormone excess or deficiency and have a direct impact on visual functioning depending on tumor type, size and (c) 2019 European Society of Endocrinology Printed in Great Britain location. Pituitary tumors also profoundly affect patients' long-term health-related quality of life (HRQoL) (3, 4, $5,6)$, which ultimately improves after treatment, but remains impaired in most patients (5). 
For most patients with a pituitary tumor, surgery is the primary treatment option. The period prior to, and shortly after, surgery is considered as a turbulent period by many patients; outcomes are typically described through clinician-reported disease parameters. These cover specific parts of the care cycle (e.g. degree of tumor resection, restoration of hormone hypersecretion (remission), recovery of visual deficits), but fail to measure to what extent the disease and treatment impact a patient's functioning and HRQoL. Therefore, it is advocated to complement these outcomes with patient-reported outcome measures (PROMS), something that has only been realized to a limited extent and to measure outcomes over the full cycle of care (primary, in- and outpatient hospital care, rehabilitation).

The current way to look at outcomes over the full cycle of care is through the framework of Value Based Health Care (VBHC). This framework, originally developed by Michael Porter and Elizabeth Teisberg, tries to increase value for the patient by improving patient-relevant outcomes and decreasing costs. According to this framework, outcomes are categorized into a three tier hierarchy, reflecting the perceived relevance for patients: (1) health status achieved or retained, (2) process of recovery and (3) sustainability of health (7). Measuring outcomes through these three tiers will assist decision making and expectation management to add optimal value to the patient and can potentially be used to alter care trajectories depending on the somatic and psychosocial needs of patients.

In order to define value, proper outcome measurement is necessary. For patients with pituitary tumors, however, the use of outcomes across all three tiers, and in particular, the use of perioperative PROMS is limited $(8,9,10,11$, $12,13,14,15,16,17)$. This may be because the routine application of these outcome measures is considered to be complicated, time consuming and too costly. Because there is no consensus yet on a core outcome set for patients with pituitary tumors the aim of the present study was to focus on outcomes first and explore the feasibility of this approach, by measuring short- to mid-term treatment outcomes of patients with various types of pituitary tumors using a comprehensive framework, the three tier framework of VHBC, alongside a well-defined surgical care pathway.

\section{Patients and methods}

\section{Study design}

This prospective longitudinal study among a cohort of consecutive patients operated for a pituitary tumor was performed between August 2016 and December 2018. The study was approved by the Ethical Committee of the Leiden University Medical Center prior to the study (p16.091).

\section{Study setting and population}

This study was conducted at a tertiary referral center, the Leiden University Medical Center (LUMC) in Leiden. The multidisciplinary treatment process was defined within a care pathway, which was specifically tailored to fit the concepts of VHBC. All consecutive patients presenting at our referral center with a pituitary tumor, older than 18 years of age, with sufficient Dutch language skills and an indication for endoscopic transsphenoidal resection of a pituitary tumor were invited to participate. Eligible patients were invited by a written letter to participate in this study by their treating endocrinologist or neurosurgeon. Consent was obtained from each patient after full explanation of the purpose and nature of all procedures used. After obtaining informed consent, patients were enrolled, and a series of questionnaires was sent at set time points.

\section{Assessments}

Outcomes were prospectively collected and are presented according to the three-tier VHBC framework. The outcome measures were selected based on their representation of the three tiers of the VBHC model and their relevance for patients (Fig. 1 and below). Questionnaires were sent or given to patients prior to surgery and at 2 days, 5 days, 6 weeks and 6 months after surgery (unless the time period the questionnaire reflects over, overlapped with the previous measurement). Time points were chosen to reflect relevant time points of the care trajectory (i.e. date of discharge or in combination with outpatient clinic appointments) and to reflect the outcomes of the tiers. This also depended on the construct a questionnaire referred to. In case of symptoms over a certain time period, they were not repeated when these periods overlapped. More specifically, the measurement on POD2 and POD5 were focused on early postoperative symptoms. Questionnaires could be filled independent of the location of the patient at the various time points and could be done either digitally or on paper, both shown to provide equivalent results (18). Detailed information on each individual questionnaire is presented in Supplementary Table 1 (see section on supplementary data given at the end of this article) and below. 




\section{Figure 1}

Disease-specific three tier model of the VBHC model for patients with a pituitary.

\section{Feasibility}

Participation was defined as the proportion of invited patients providing informed consent and completing at least one questionnaire. Retention was defined as the proportion of participants who completed the questionnaire and/or were seen at the outpatient clinic at 6 months. Response rate was calculated as the proportion of patients who returned at least one questionnaire. The number of missing items per questionnaire and per time-point were also calculated.

\section{Baseline patient characteristics}

This included age, sex, marital status, level of education, comorbidities, tumor type, date of diagnosis, pituitary function, visual functioning and cerebral nerve deficits.
Marital status was categorized into married/living together or not (alone, divorced, widow). Level of education was categorized into low, intermediate or high, based on the guidelines of Statistics Netherlands (CBS) (19), which correspond with the International Standard Classification of Education Fields of Training and Education 2013 of the UNESCO (20). Comorbidities were assessed with the Dutch comorbidity questionnaire, Statistics Netherlands, which assesses the most common chronic diseases and disorders in the Netherlands (21) and were categorized into diabetes mellitus, neurovascular, cardiovascular and malignancies. Tumor type was divided into (1) non-functioning pituitary adenoma (NFA), (2) acromegaly (ACRO), (3) Cushing's disease (CD), (4) prolactinoma (PRL), (5) Rathke's cleft cyst (RCC), (6) craniopharyngioma (cranio). 
Pituitary function was defined as (1) no deficits, (2) single hormone deficiency, (3) multiple hormone deficiencies, (4) multiple hormone deficiencies plus diabetes insipidus (DI) and (5) DI alone. Visual functioning was defined as (1) no deficits, (2) quadrant anopia or less (mild) and (3) hemianopia (severe). Prior treatment was classified into four categories: (1) no treatment, (2) prior medical (tumor) treatment, (3) prior surgery and (4) prior radiotherapy. A detailed description of the treatment algorithm, which was in line with existing guidelines, has previously been published $(22,23,24)$. In addition, all patients, including patients with $\mathrm{CD}$, received perioperative hydrocortisone, according to a standard protocol, regardless of tumor type. Hydrocortisone was discontinued only after confirmation of recovery of the endogenous pituitary-adrenal axis or persistent disease.

\section{Tier 1: health status achieved or retained}

Tier 1 represents the core outcomes at the endpoint of treatment, which for this study was assessed at 6 months after surgery. It included survival (yes/no), endocrine remission among patients with functioning tumors (complete/partial/no), visual recovery in those with visual impairments (visual field/acuity) (complete/ partial/no recovery/worsening), recovery of pituitary function in those with hypopituitarism (complete/ partial/no recovery/worsening) and degree of resection (complete/partial/unsure). Endocrine remission was defined as normalized overproduction without medication. Recovery of patient-reported outcomes was defined as the minimal important change, a clinically relevant change between baseline and 6 months and calculated per patient per questionnaire as half a standard deviation (SD) difference between the two time points (25). Depending on the magnitude of the change, this was categorized into improved (>0.5 S.D.), deteriorated (>0.5 s.D.) or no important change (<0.5 s.D.). Recovery of disease burden was measured through a modified version of the (Leiden Bother and Needs Questionnairepituitary (LBNQ-pituitary)) (26), which was modified in order to make it suitable for repeated measurements. It ranges from 0 to 100, and higher scores indicate a greater disease burden or need for help and was not assessed on POD2. Recovery of HRQoL was measured with the short form-36 (SF-36), which ranges from 0 to 100 and was not assessed on POD2 and POD5. Higher scores for physical or mental functioning indicate better HRQoL (27). Recovery of utility (EQ-index, scale anchored at 0 (as bad as death) and 1 (perfect health)) and self-reported health status (EQ-VAS, range 0 to 100) were measured through the EuroQoL (EQ-5D-5L, Dutch Tariff) $(28,29)$. Higher scores indicate a better perceived health status. Visual functioning was assessed through the VFQ-25 (range 0 to $100)$, and higher scores indicate better visual functioning. The VFQ-25 was not assessed on POD2 (30).

\section{Tier 2: process of recovery}

This concerns the course of outcomes over time and consists of time to recovery of disease burden, HRQoL, utility and visual function (as measured through the VFQ25). Recovery was defined as a significant improvement over time compared to baseline (mean change). Furthermore, time to return to work (short form-health and labor questionnaire (SF-HLQ)) was determined as change between baseline and 6 months (31).

Disutility of care or treatment process included length of stay, nasal morbidity over time (anterior skull base nasal inventory-12 (ASK nasal-12) (32)/sino-nasal outcome test22 (SNOT-22)) (33) and complications (readmission $<30$ days, endocrine complications: transient DI/permanent DI/delayed hyponatremia/new pituitary deficiencies, neurosurgical complications: postoperative CSF leak/ severe epistaxis requiring surgery/mild epistaxis/bleedings or other). For both the ASK-12 (range 0-5) and the SNOT22 (range 0-110), higher scores indicate worse nasal functioning. The ASK-12 and SNOT-22 were not assessed on POD2.

\section{Tier 3: sustainability of health}

This concerns outcomes up to 6 months after surgical treatment, mid- to long-term effects of treatment, included new onset of permanent pituitary deficiencies, accompanying replacement therapies, additional treatments, permanent complications (of intervention and disease) and recurrences necessitating additional treatment.

\section{Statistical analysis}

Data entry and control were performed through an online survey platform (NETQ, NETQ Healthcare B.V., Utrecht, The Netherlands). All statistical analyses were performed with SPSS 25.0 software (SPSS Inc.). Nominal variables are presented as frequencies with percentages, numerical variables as means and S.D. or medians with interquartile ranges (IQR), and comparisons between tumor types were performed through the KruskalWallis test, where applicable. Categorical variables 
were calculated as frequencies with percentages and comparisons were performed through chi-square analyses and Fisher's exact test, where applicable.

Due to differences in surgical indications and surgical goals between tumor types, only descriptive statistics were presented for clinician-reported outcome measures. Because PROMS measure different constructs, PROMS were compared between tumor groups. Differences between tumor types are presented for groups larger than ten patients. Longitudinal analysis was performed via general estimating equations (GEEs) analysis and results are presented as means with corresponding 95\% confidence intervals (CIs). For all analyses, the level of significance was set at $P<0.05$ (two-sided). Missing data on the questionnaires were handled by parcel summary imputation (34).

\section{Results}

A total of 142 patients with a pituitary tumor and an indication for surgery were seen at the outpatient clinic between September 2016 and July 2018, of which 103 patients $(73 \%)$ decided to participate in this study. Of those not participating, 11 declined participation (8\%). Other reasons for non-participation were emergency surgery $(n=10,7 \%)$, age $<18$ years $(n=4,3 \%)$, cognitive impairments $(n=7,5 \%)$, language barrier $(n=4,3 \%)$ and preoperative suspicion of other pathology $(n=3,2 \%)$.

All patients underwent endoscopic transsphenoidal surgery within the study period and at the end of follow-up all patients were retained. Of those, 103 completed questionnaires at baseline (100\%), 101 at day 2 (98\%), 100 at day 5 (97\%), 99 at 6 weeks (96\%) and 99 at 6 months (96\%). The number of missing items per questionnaire and per time-point is described in Supplementary Table 2 and ranges between 0.1 and $8.0 \%$.

\section{Baseline characteristics}

The median age of included patients was 53 (IQR 37-65) years, and 64 patients (62\%) were female. The largest proportion of patients had been diagnosed with an NFA ( $n=47,46 \%$ ), followed by 45 patients with functioning tumors (44\%, range $14-16 \%$ per tumor type) and 11 patients (11\%) with other pituitary region tumors (six RCC and five cranio). Preoperative hormone deficiencies were present in 50 patients (49\%), and 47 patients (46\%) had visual deficits. For most patients the surgical treatment was the primary surgical intervention (85\%) (Table 1).

\section{Tier 1: health status achieved or retained (after 6 months)}

Survival

There was no mortality (Table 2).

\section{Degree of health or recovery}

Endocrine remission Of the 45 patients with functioning tumors, 31 (69\%) were in endocrine remission after 6 months, which was the highest among patients with CD: $80 \%(n=12)$, followed by patients with a PRL: $75 \%(n=12)$ and ACRO: $50 \%(n=7)$ (Table 2).

Visual recovery There were 47 patients with preoperative visual deficits, of which the majority with an NFA $(n=33,70 \%)$. Complete visual recovery was achieved among 21 patients (46\%), partial recovery in $24(52 \%)$, whereas only 1 patient (2\%) did not improve after surgery (Table 2).

Recovery of pituitary function Of the 50 patients with preoperative pituitary deficiencies (49\%), one or more pituitary axes recovered in 14 patients (28\%) after surgery. New onset of hypopituitarism occurred in 12 patients (12\% of total cohort) (Table 2 ).

Degree of resection Overall, complete resection was achieved in 72 patients $(70 \%)$, with the highest rate seen among patients with PRLs $(n=12,75 \%)$, followed by patients with an NFA $(n=34,72 \%)$, ACRO $(n=10,71 \%)$ and CD $(n=9,60 \%)$. Degree of resection did not always align with the, more relevant, endocrine results among patients with functioning tumors. For example, a possible radiological residual could not be excluded among two patients despite being in endocrine remission (e.g. normalization of hormone excess). Also, among five patients with persisting endocrine disease (e.g. overproduction), there was no radiological residual present (Table 2).

\section{Self-perceived recovery}

LBNQ pituitary In total, at 6 months, 42 patients (41\%) reported a clinically relevant improvement of their overall disease burden. Clinically relevant deterioration occurred in 12 patients $(12 \%)$. There was a distinct difference between the various tumor types, which is best illustrated by the overall disease burden of patients with a PRL 


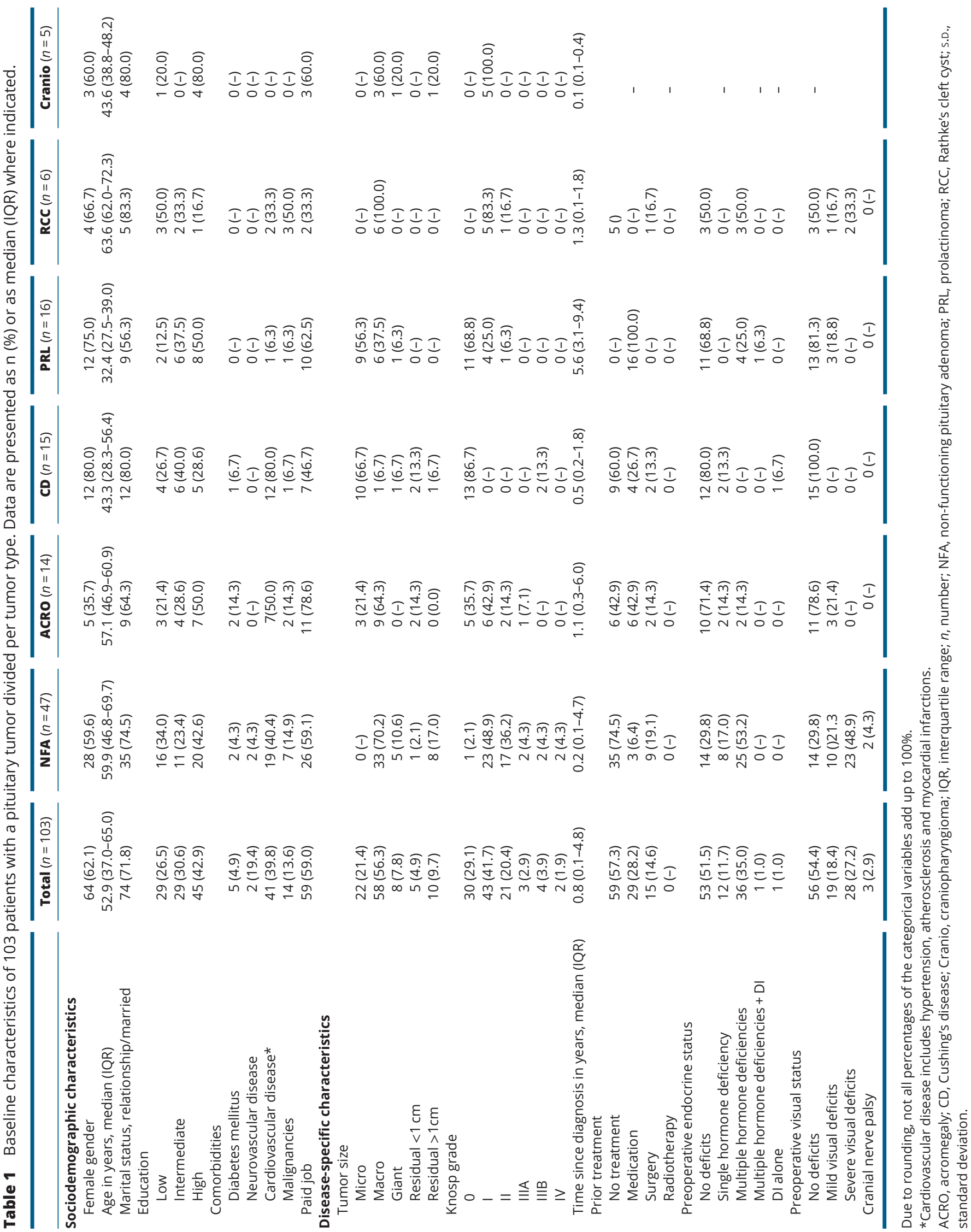


(Fig. 2A). These patients, usually refractory or intolerant to dopamine agonists, have comparable disease burden as patients with $\mathrm{CD}$ at baseline (mean difference 8.3, 95\% CI -21.7 to $5.0, P=.22$ (CD vs PRL)), which is significantly worse compared to patients with an NFA (mean difference 27.1, 95\% CI 16.1-38.1, $P<.001$ (PRL vs NFA) or ACRO (mean difference 26.5, 95\% CI 14.6-38.3, $P<.001$ (PRL vs ACRO)). Patients with CD also had significantly worse scores compared to NFA (mean difference 18.8, 95\% CI 9.8-27.7, $P<.001$ (CD vs NFA)) and ACRO (mean difference 18.1, 95\% CI 8.3-27.9, $P<.001$ (CD vs ACRO)). Patients with a PRL, however, improved significantly after surgery (mean improvement 19.1, 95\% CI 12.1-26.2, $P<.001$ ), while patients with CD did not (mean improvement $6.8,95 \% \mathrm{CI}-4.1$ to $17.7, P=.22$ ). Patients with ACRO are comparable to those with an NFA both at baseline (mean difference $0.6,95 \% \mathrm{CI}-3.3$ to $5.9, P=.85$ ), and 6 months after surgery (significant mean improvement $4.2,95 \%$ CI $0.03-8.3, P=.05$ (NFA), respectively 5.8, 95\% CI 0.3-11.3, $P=.04($ ACRO) $)$.

SF-36 Concerning HRQoL, relatively more patients improved on the mental aspect of HRQoL $(n=42,51 \%)$, than on the physical $(n=35,34 \%)$, while there were no differences between tumor types.

EQ-5D Utility, as measured by the EQ index, improved among 29 patients (28\%), most among patients with a PRL $(n=9,56 \%)$. Self-perceived health status improved among 33 patients (32\%).

VFQ-25 Among those with preoperative visual deficits, self-reported visual functioning improved in 30 patients (64\%).

\section{Tier 2: process of recovery (0-6 months)}

Time to recovery and return to normal activities

LBNQ pituitary Regarding the course of recovery, the overall perceived disease burden improved significantly 5 days after surgery for all tumor types and remained improved in all tumor types, except for patients with $\mathrm{CD}$, who returned back to baseline from 6 weeks onward (Fig. 2A).

SF-36 With regard to HRQoL, mental functioning improved significantly 6 weeks after surgery among patients with an NFA (mean improvement 4.7, 95\% CI
1.5-7.8, $P=.004$ ), or a PRL (mean improvement 9.4, 95\% CI 6.3-12.6, $P=.002$ ) and remained improved in both at 6 months (Fig. 2B). Physical functioning significantly deteriorated after 6 weeks for patients with an NFA (mean deterioration 4.1, 95\% CI 1.6-6.5, $P=.001$ ) and returned to baseline at 6 months (mean deterioration 1.1, 95\% CI -1.9 to $4.2, P=.46$ ). Patients with ACRO had significantly better physical functioning at 6 weeks (mean improvement 6.6, 95\% CI 3.2-10.0, $P<.001$ ), which remained improved at 6 months (mean improvement 6.2, 95\% CI 1.8-10.6, $P=.005$ ) (Fig. 2C).

EQ-5D Utility deteriorated significantly 2 days after surgery among patients with an NFA (mean deterioration $0.05,0.01-0.09, P=.02$ ) and CD (mean deterioration 0.11, 95\% CI 0.02-0.19, $P=.01$ ), however, returned to baseline at 5 days after surgery and remained unchanged during follow-up. Patients with a PRL improved significantly after 6 months (mean improvement 0.07, 95\% CI $0.02-$ $0.12, P=.01$ ) (Fig. 2D and E).

VFQ-25 Among patients with visual deficits, self-perceived visual functioning improved significantly 5 days after surgery compared to baseline (mean improvement 13.4, 95\% CI 5.5-21.3, $P=.001$ (mild deficits) and 9.3, 95\% CI 5.3-13.4, $P<.001$ (severe deficits)), and improved further during the course of follow-up (mean improvement at 6 months 21.1, 95\% CI 11.7-30.6, $P<.001$ (mild deficits), 12.5, 95\% CI 8.0-17.0, $P<.001$ (severe deficits)) (Fig. 2F).

Return to work Of the 56 patients with a paid job prior to surgery, $51(86 \%)$ still had a paid job after 6 months (Table 3).

\section{Disutility of care or treatment process}

Length of stay The median length of hospital stay after surgery was 3 days (IQR 2-5), which was longest among patients with CD, shortest among patients with an NFA, PRL or ACRO and was significantly different between tumor types $(P<.001)$ (Table 3$)$.

Nasal morbidity For all tumor types, postoperative nasal functioning as measured by the ASK nasal-12 deteriorated significantly 5 days after surgery compared to baseline (mean difference range 0.9-1.7) and remained significantly worse compared to baseline up to 6 weeks 
Table 2 Tier 1: health status achieved or retained at 6 months among 103 surgically treated patients with a pituitary tumor stratified according to tumor type.

\begin{tabular}{l}
\hline \\
\hline Survival, $n$ (\%) \\
Degree of resection, $n$ (\%) \\
Complete resection \\
Residual \\
Unsure \\
No MRI \\
Endocrine remission of hormone excess \\
Remission \\
Partial recovery \\
Persistent disease \\
Pituitary function, $n$ (\%)* \\
Complete recovery \\
Partial recovery \\
Persistent deficits \\
Deteriorated \\
Visual outcome, $n$ (\%) \\
Complete recovery \\
Partial recovery \\
Persistent deficits/acuity \\
Deteriorated \\
LBNQ-pituitary total score, $n$ (\%) \\
Improved \\
Not importantly changed \\
Deteriorated \\
SF-36 MCS, $n$ (\%) \\
Improved \\
Not importantly changed \\
Deteriorated \\
SF-36 PCS, $n$ (\%) \\
Improved \\
Not importantly changed \\
Deteriorated \\
EQ index, $n$ (\%) \\
Improved \\
Not importantly changed \\
Dot importantly changed \\
Deteriorated \\
Improved \\
Dot importantly changed \\
\hline
\end{tabular}

\begin{tabular}{c}
\hline Total $(n=$ \\
\hline $0(-)$ \\
$72(69.9)$ \\
$20(19.4)$ \\
$6(5.8)$ \\
$5(4.9)$
\end{tabular}

s, $n(\%)^{+}$

$31(68.9)$

$11(24.4)$

3 (6.7)

7 (11.9)

7 (11.9)

$33(55.9)$

$12(20.3)$

$21(45.7)$

$24(52.2)$

$1(2.2)$

$0(-)$

$42(40.8)$

49 (47.6)

$12(11.7)$

$42(40.8)$

$45(43.7)$

16 (15.5)

$35(34.0)$

45 (43.7)

$23(22.3)$

29 (28.2)

$53(51.5)$

20 (19.4)

$33(32.0)$

$35(34.0)$

34 (33.0)

$30(63.8)$
$14(29.8)$
$3(6.4)$

\begin{tabular}{c}
\hline NFA $(n=47)$ \\
\hline $0(-)$ \\
$34(72.3)$ \\
$10(21.3)$ \\
$3(6.4)$ \\
$0(-)$ \\
- \\
- \\
-
\end{tabular}

ACRO

$(n=14)$

$4(10.5)$

4 (10.5)

24 (63.2)

$6(15.8)$

$13(40.6)$

18 (56.3)

1 (3.1)

0 (-)

16 (34.0)

24 (51.1)

7 (14.9)

$22(46.8)$

20 (42.6)

5 (10.6)

10 (21.3)

22 (46.8)

15 (31.9)

$10(21.3)$

28 (59.6)

9 (19.1)

13 (27.7)

$17(36.2)$

$17(36.2)$

$22(66.7)$

9 (27.3)

2 (6.1)

$10(71.4)$

3 (21.4)

1 (7.7)

$0(-)$

7 (50.0)

7 (50.0)

0 (-)

3 (50.0)

1 (16.7)

2 (33.3)

$0(-)$

2 (66.7)

1 (33.3)

$0(-)$

$0(-)$

3 (21.4)

11 (78.6)

$0(-)$

2 (14.3)

8 (57.1)

4 (28.6)

9 (64.3)

4 (28.6)

1 (7.1)

4 (28.6)

7 (50.0)

3 (21.4)

3 (21.4)

6 (42.9)

4 (28.6)

$1(7.1)$
$2(14.3)$
$0(-)$

$0(-) \quad 0(-)$

PRL $(n=16)$

$\overline{\operatorname{RCC}(n=6)}$

$\frac{\overline{C r a n i o}(n=5)}{0(-)}$

$9(60.0)$

2 (13.3)

1 (6.7)

3 (20.0)

12 (75.0)

1 (6.3)

1 (6.3)

2 (12.5)

4 (66.7)

2 (33.3)

$0(-)$

$0(-)$

3 (60.0)

$12(80.0)$

0 (-)

$3(20.0)$

12 (75.0)

$4(25.0)$

0()

0()

4 ()

1 ()

0 (-)

0 (-)

0 (-)

$0(-)$

$6(40.0)$

$6(40.0)$

3 (20.0)

5 (33.3)

5 (33.3)

5 (33.3)

$6(40.0)$

$6(40.0)$

0 (-)

3 (20.0)

0 ()

0 ()

$3(100.0)$

0 ()

-

-

2 (66.7)

1 (33.3)

0 (-)

0 (-)

0 ()

2 (66.7)

0 ()

1 (33.3)

2 (40.0)

$0(-)$

$0(-)$

1 (33.3)

2 (66.7)

$0(-)$

$0(-)$

$13(81.3)$

$3(50.0)$

3 (18.8)

3 (50.0)

0 (-)

0 (-)

9 (56.3)

3 (50.0)

6 (37.5)

3 (50.0)

$0(-)$

1 (6.3)

2 (13.3)

6 (37.5)

1 (16.7)

9 (56.3)

$4(66.7)$

1 (6.3)

1 (16.7)

$0(-)$

$6(40.0)$

9 (56.3)

2 (33.3)

2 (40.0)

$6(40.0)$

3 (20.0)

6 (37.5)

$3(50.0)$

2 (40.0)

$1(6.3)$

1 (16.7)

$3(60.0)$

4 (26.7)

8 (50.0)

3 (50.0)

6 (37.5)

2 (33.3)

2 (12.5)

1 (16.7)

$3(60.0)$

8 (53.3)

$0(-)$
$0(-)$
$0(-)$

$2(66.7)$
$1(33.3)$
$0(-)$

2 (66.7)

2 (40.0)

For patient-reported outcome measures improvement/deterioration indicates a change $>1 / 2 \mathrm{SD}$.

${ }^{+{ }^{\top} \mathrm{T}}$ Among those with preoperative deficits/hormone excess (except for deterioration).

ACRO, acromegaly; CD, Cushing's disease; Cranio, craniopharyngioma; EQ, EuroQoL; IQR, interquartile range; LBNQ-Pituitary, Leiden Bother and Needs Questionnaire - Pituitary; $n$, number; NFA, non-functioning pituitary adenoma; PRL, prolactinoma; RCC, Rathke's cleft cyst; SD, standard deviation; SF-36, Short Form-36; VAS, visual analog scale; VFQ-25, Visual functioning Questionnaire-25.

after surgery. Patients with ACRO or a PRL recovered quicker and patients did not have significantly different nasal morbidity compared to baseline at 6 weeks (mean difference $0.3,95 \%$ CI -0.8 to $0.1, P=.14$ (ACRO), 0.4 , $95 \% \mathrm{CI}-.02$ to $1.0, P=.19$ (PRL)). At 6 months, 24 patients (23\%) had a clinically relevant deterioration as measured by the ASK nasal-12 (Table 3), and the mean difference ranged between -0.4 (ACRO) and 0.2 (CD) (Fig. 3).

Complications Neurosurgical complications were present in 19 patients (18\%). Most complications did not have long-term implications; however, four patients had major 

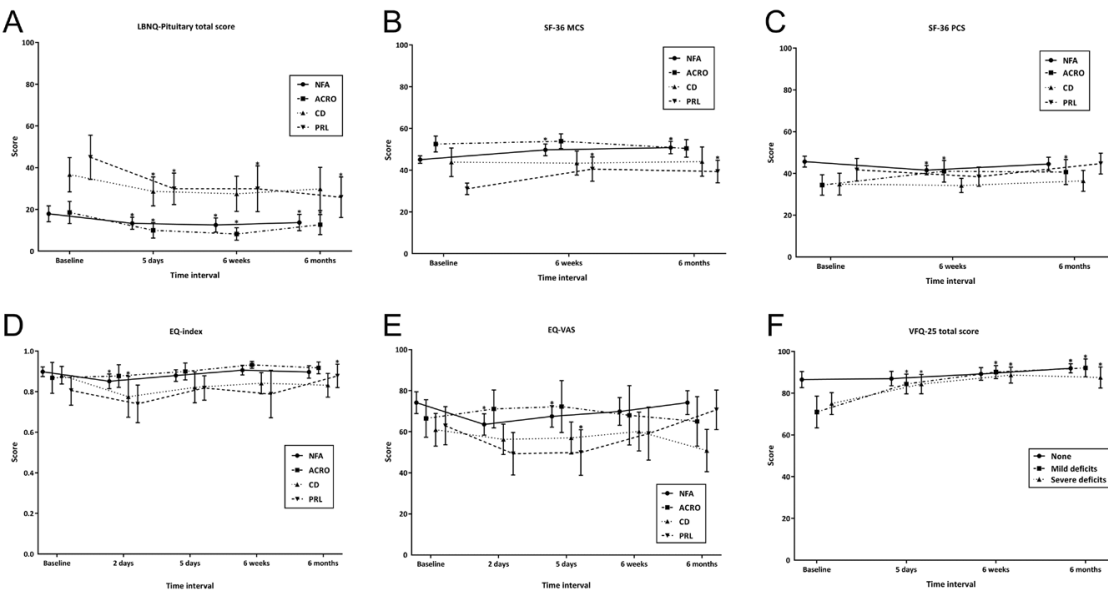

\section{Figure 2}

Perioperative patient-reported outcomes over time per tumor type. (A) Higher scores indicate worse disease burden. (B, C, D, E and F) Higher scores indicate better health-related quality of life, utility, self-perceived health status, visual functioning. *Significant change compared to baseline (within tumor type). ACRO, acromegaly; $C D$, Cushing's disease; $E Q$, EuroQoL; LBNQ-Pituitary, Leiden Bother and Needs Questionnaire - Pituitary; NFA, non-functioning pituitary adenoma; PRL, prolactinoma; SF-36, Short Form-36; VAS, visual analog scale; VFQ-25, Visual

Functioning Questionnaire-25. complications after surgery, which typically occurred after more complex surgeries (one patient with an NFA had acute sudden deafness after a CSF leak, one patient had a postoperative hemiplegia due to multiple major intracranial hemorrhages after resection of a giant NFA and two patients had thalamic infarctions after resection of giant tumors (one NFA/1 craniopharyngioma)). Endocrinological complications occurred in 41 patients $(40 \%)$, which were mostly of transient nature $(n=28$, 68\%) (Table 3).

\section{Tier 3: Sustainability of health}

Long-term consequences of therapy

Among the 45 patients with functioning tumors, unchanged disease activity was present in 3 patients (7\%), all patients with CD. During the course of the study there were no recurrences (up to 6 months after treatment) in patients in remission. New onset of pituitary hormone deficiency occurred in 12 patients (12\%), most frequently of the corticotropic axis. In total, 52 patients $(50 \%)$ had one or more pituitary deficiencies at the end of follow-up (Table 4).

\section{Discussion}

This prospective cohort study shows that the outcomes of surgery for patients with various pituitary tumors can be well reflected using a comprehensive set of clinical and patient-reported outcomes. In general, improvement of outcomes over the full cycle of care was seen after surgery, but health status at 6 months, the process of recovery and mid- to long-term outcomes (although only partially measured) were highly variable between individuals, which is partly explained by tumor type.

While pituitary tumor surgery is usually performed with the goal to increase value for the patient, health outcomes are often primarily measured through clinician-reported outcomes, such as recovery of vision or remission of hypersecretion, rather than combining them with patient-reported outcomes (PROs). Although we are aware that long-term outcomes presented in this study can only serve as a proxy of the actual long-term outcomes, to the best of our knowledge this study is the first to describe a comprehensive set of outcomes over time for patients with a pituitary tumor through the framework of VBHC and therefore serves as a benchmark for future studies. New insights into the perioperative development of disease burden and HRQoL over time give a better understanding of the disease course and will enable shared decision making and expectation management based on perioperative outcomes in patients with different treatment options and tumor types.

Regarding disease burden, we report here for the first time the perioperative burden of disease among patients with pituitary tumors. These outcomes, in combination with the self-perceived needs for support, will enable clinicians to individualize care trajectories.

With regard to HRQoL, the limited number of studies that do report results, show an increase in HRQoL after surgical intervention compared to preoperative outcomes (as measured by the SF-36) (8, 12, 13, 14, 15, $17,35)$. The interpretability of these results for clinical practice, however, is difficult since these questions focus 
Table 3 Tier 2: Process of recovery among 103 surgically treated patients with a pituitary tumor stratified according to tumor type. Data are presented as $\mathrm{n}(\%)$ or as median (IQR) where indicated.

\begin{tabular}{|c|c|c|c|c|c|c|c|}
\hline & Total $(n=103)$ & NFA $(n=47)$ & ACRO $(n=14)$ & $\mathbf{C D}(n=15)$ & $\mathbf{P R \mathbf { L }}(n=16)$ & $\operatorname{RCC}(n=6)$ & Cranio $(n=5)$ \\
\hline Length of stay, median (IQR) & $3.0(2-5)$ & $3.0(2-5)$ & $3.0(3-4)$ & $5.0(4-7)$ & $3.0(2-5)$ & $2.0(2-3)$ & $12.0(7-12)$ \\
\hline Return to work & $51(86.4)$ & $23(88.5)$ & $9(81.8)$ & $6(85.7)$ & $10(100.0)$ & $2(100.0)$ & $1(33.3)$ \\
\hline \multicolumn{8}{|l|}{ ASK-12 } \\
\hline Improved & 20 (19.4) & 7 (14.9) & 5 (35.7) & $0(-)$ & 5 (31.3) & 2 (33.3) & $1(20.0)$ \\
\hline Not importantly changed & $57(55.3)$ & $30(63.8)$ & $6(42.9)$ & $9(60.0)$ & $6(37.5)$ & $4(66.7)$ & $2(40.0)$ \\
\hline Deteriorated & $24(23.3)$ & $10(21.3)$ & $2(14.3)$ & $6(40.0)$ & $4(25.0)$ & $0(-)$ & $2(40.0)$ \\
\hline \multicolumn{8}{|l|}{ SNOT-22 } \\
\hline Improved & 37 (35.9) & $10(21.3)$ & $9(64.3)$ & $5(33.3)$ & $8(50.0)$ & $2(33.3)$ & $3(60.0)$ \\
\hline Not importantly changed & $50(48.5)$ & $28(59.6)$ & $4(28.6)$ & $6(40.0)$ & $7(43.8)$ & $3(50.0)$ & $2(40.0)$ \\
\hline Deteriorated & $16(15.5)$ & $9(19.1)$ & $1(7.1)$ & $4(26.7)$ & $1(6.3)$ & $1(16.7)$ & $0(-)$ \\
\hline \multicolumn{8}{|l|}{ Complications } \\
\hline Readmission & $13(12.6)$ & $7(14.9)$ & $2(14.3)$ & $0(-)$ & $2(12.5)$ & $1(16.7)$ & $1(20.0)$ \\
\hline Any complication & $50(48.5)$ & $20(42.6)$ & $5(35.7)$ & $8(53.3)$ & $10(62.5)$ & $2(33.3)$ & $5(100.0)$ \\
\hline Transient DI, & $27(26.2)$ & $13(27.7)$ & $2(14.3)$ & $5(33.3)$ & $7(43.8)$ & $0(-)$ & $0(-)$ \\
\hline Permanent DI, & $8(7.8)$ & $1(2.1)$ & $1(7.1)$ & $1(6.7)$ & $0(-)$ & $1(16.7)$ & $4(80.0)$ \\
\hline Delayed hyponatremia, & $13(12.6)$ & $7(14.9)$ & $1(7.1)$ & $1(6.7)$ & $1(6.3)$ & $1(16.7)$ & $2(40.0)$ \\
\hline $\begin{array}{l}\text { New onset pituitary } \\
\text { deficiency }\end{array}$ & $12(11.7)$ & $6(12.8)$ & $0(-)$ & $1(6.7)$ & $0(-)$ & $1(16.7)$ & $4(80.0)$ \\
\hline Postoperative CSF leak, & $7(6.8)$ & $2(4.3)$ & $0(-)$ & $0(-)$ & $1(6.3)$ & $1(16.7)$ & $2(40.0)$ \\
\hline $\begin{array}{l}\text { Severe epistaxis requiring } \\
\text { surgery }\end{array}$ & $2(1.9)$ & $0(-)$ & $0(-)$ & $0(-)$ & $2(12.5)$ & $0(-)$ & $0(-)$ \\
\hline Mild epistaxis, & $5(4.9)$ & $2(4.3)$ & $2(14.3)$ & $1(6.7)$ & $0(-)$ & $0(-)$ & $0(-)$ \\
\hline Other* & $4(3.9)$ & $2(4.3)$ & $0(-)$ & $0(-)$ & $0(-)$ & 1 (16.7) & $1(20.0)$ \\
\hline
\end{tabular}

IQR, interquartile range; NFA, non-functioning pituitary adenoma; ACRO, acromegaly; CD, Cushing's disease; PRL, prolactinoma; RCC, Rathke's cleft cyst; Cranio, craniopharyngioma; DI, diabetes insipidus; CSF, cerebrospinal fluid; ASK nasal-12, anterior skullbase nasal inventory; SNOT-22, Sino-nasal outcome test.

*thalamic infarction, rebleed, acute sudden deafness.

on more general constructs instead of disease-specific issues. Although the design of the study did not allow for cause-effect relations, we hypothesize that some of the observed outcomes can be explained by the case mix of our tertial referral center, and preoperative severity of disease burden. We have a relatively high number of referrals of prolactinoma with drug intolerance, which likely negatively affects disease burden. Moreover, the timing of return to normal daily life activities frequently occurs between 6 weeks and 6 months. The perception of disease burden in that period in some patients may be negatively influenced by the confrontation of having limited energy, whereas the demands of daily life are increasing. This might in part explain why physical HRQoL deteriorated among patients with NFAs and improved among patients with ACRO.

Visual QoL was previously reported to improve after treatment (13), which was in line with results from our study. Regarding nasal morbidity, previous studies have also shown an initial increase in nasal morbidity, and most have shown restoration back to baseline after 6 months to 1 year after treatment $(9,10,11,14,15)$, which is also in line with our findings (Table 3 and Supplementary Table 3).

\section{Strengths, limitations and future perspectives}

One of the main strengths of this study is the high participation rate and low amount of missing data. We are aware that it takes effort to motivate patients to fill out questionnaires, however, with a good Information Technology (IT) infrastructure and when feedback of results is given to patients, we believe these results can
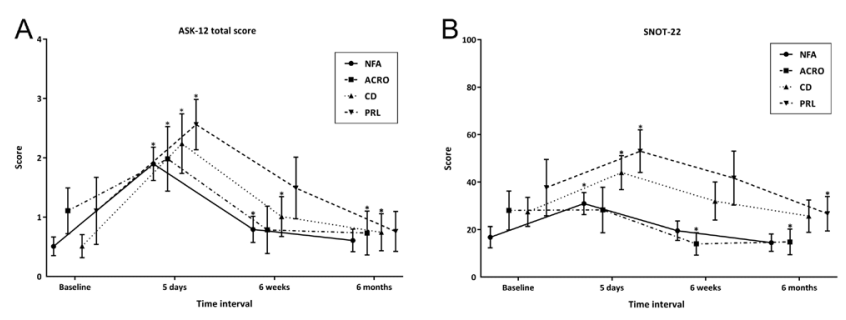

\section{Figure 3}

Perioperative patient-reported nasal functioning over time per tumor type. (A and B) Higher scores indicate worse nasal functioning burden. * Significant change compared to baseline (within tumor type). ACRO, acromegaly; ASK nasal-12, anterior skullbase nasal inventory; CD, Cushing's disease; NFA, non-functioning pituitary adenoma; PRL, prolactinoma; SNOT-22, sino-nasal outcome test. 
Table 4 Tier 3: Sustainability of health. Data are presented as n (\%).

\begin{tabular}{|c|c|c|c|c|c|c|c|}
\hline & Total $(n=103)$ & NFA $(n=47)$ & ACRO $(n=14)$ & $\mathbf{C D}(n=15)$ & $\mathbf{P R \mathbf { L }}(n=16)$ & $\mathbf{R C C}(n=6)$ & Cranio $(n=5)$ \\
\hline $\begin{array}{l}\text { Reoperation }>30 \text { days and }<6 \\
\text { months }\end{array}$ & $0(-)$ & $0(-)$ & $0(-)$ & $0(-)$ & $0(-)$ & $0(-)$ & $0(-)$ \\
\hline $\begin{array}{l}\text { Chronic supplementation of } \\
\text { pituitary deficits }\end{array}$ & $52(50.5)$ & $34(72.3)$ & $3(21.4)$ & $5(33.3)$ & $3(18.8)$ & $3(50.0)$ & $4(80.0)$ \\
\hline Recurrence & $0(-)$ & $0(-)$ & $0(-)$ & $0(-)$ & $0(-)$ & $0(-)$ & $0(-)$ \\
\hline Persistent hormone excess & $3(6.7)$ & - & $0(-)$ & $3(20.0)$ & $0(-)$ & - & - \\
\hline
\end{tabular}

also be achieved in daily practice. Therefore, both patients and healthcare providers need to be aware of the benefits of PROMS, which can lead to targeted interventions at the individual, but also at the group level.

Although exact time to recovery cannot be reproduced due to the nature of this study, measurement points were chosen to represent routine clinical evaluations, making it more feasible that the PROMS will be implemented in the outpatient setting and will enable clinical decision making, for example, additional nasal examinations or referrals to rehabilitation centers, psychologists or sexologists. Due to the good experiences during this study we have continued assessment of PROMS at our institute, in a somewhat modified version with evaluation at baseline, 6 weeks and 6 months and yearly thereafter.

At the group level, it is important to expand the knowledge initiated by this study, since collective reporting of perioperative outcomes will enable comparison of outcomes between centers and initiate improvement trajectories. Furthermore, outcomes presented here can be considered for the development of a core outcome set (COS) for patients with a pituitary tumor. Traditionally, these COS were defined as those outcomes minimally necessary for the measurement and reporting in clinical trials of specific diseases (36). However, they can also be used for clinical practice purposes such as expectation management, evaluation of clinical care trajectories at the group and patient level, and for the comparison of outcomes between centers. This study contributes toward reducing the previous knowledge gap, which restricts proper selection of instruments to measure PROMS for a COS and further opens the path toward value driven healthcare.

One of the key limitations of our study is the relatively small number of patients, in particular for some of the tumor types, which not only hampers comparisons between groups with different diagnosis in our study, but also comparisons with other studies. For this reason, we were also unable to correct for potential confounders or effect modifiers, for example, tumor-related factors, such as tumor size. The short duration of follow-up is also a limitation, especially for the cases of hormone excess syndromes and recovery of hypopituitarism, specifically posterior pituitary deficiency. In clinical practice, for instance, it is well known that patients with CD often need longer recovery time after surgery (37), and this might (partially) explain the differences between tumor types found in this study. Future research should extend the duration of follow-up for the analysis of perioperative outcomes, to enable interpretability of the relationship between short- and long-term outcomes and investigate the optimal time frame to properly evaluate disease burden/HRQoL. This argument also holds for remission rates. Tumor types have different outcome parameters of interest, for example endocrine remission and radiological outcome, and differences between tumor types might to some extent be explained by treatment by differences in surgical goals (treatment by indication), where optical preservation is often the goal of surgery for patients with an NFA, remission/complete resection is often the goal for functioning tumors.

Finally, to further elaborate on the value for the patient alongside the framework of $\mathrm{VBHC}$, it is also necessary to also evaluate costs of treatment, as value can be increased by improving outcomes, but also by lowering costs. Assessing costs and expanding the assessment of outcomes are necessary in order to define value for the patient.

Further expansion of knowledge alongside the comprehensive set of outcomes used in this study show promising opportunities, which might lead to the identification of unexplained differences between individual patients and impairments in HRQoL.

\section{Conclusion}

The impact of a surgical intervention for patients with pituitary tumors can be well reflected through a comprehensive set of clinician-reported measurements in 
combination with PROMS. Measuring outcomes appears to be feasible. Disease-specific questionnaires in particular are able to show differences in the disease course between various tumor types. This information can help clinicians to more accurately inform patients about the expected outcome and improve the process of recovery and personalize care.

\section{Supplementary data}

This is linked to the online version of the paper at https://doi.org/10.1530/ EJE-19-0344.

\section{Declaration of interest}

The authors declare that there is no conflict of interest that could be perceived as prejudicing the impartiality of this study.

\section{Funding}

This research did not receive any specific grant from any funding agency in the public, commercial or not-for-profit sector.

\section{Acknowledgements}

The authors would like to acknowledge Dr E van Zwet for his valuable input for the data analysis. W R van Furth and N R Biermasz: Shared last author.

\section{References}

1 Karavitaki N. Prevalence and incidence of pituitary adenomas. Annales d'Endocrinologie 201273 79-80. (https://doi.org/10.1016/j. ando.2012.03.039)

2 Ostrom QT, Gittleman H, Fulop J, Liu M, Blanda R, Kromer C, Wolinsky Y, Kruchko C \& Barnholtz-Sloan JS. CBTRUS statistical report: primary brain and central nervous system tumors diagnosed in the United States in 2008-2012. Neuro-Oncology 201517 iv1-iv62. (https://doi.org/10.1093/neuonc/nov189)

3 van der Klaauw AA, Kars M, Biermasz NR, Roelfsema F, Dekkers OM, Corssmit EP, van Aken MO, Havekes B, Pereira AM, Pijl H et al. Disease-specific impairments in quality of life during long-term follow-up of patients with different pituitary adenomas. Clinical Endocrinology 200869 775-784. (https://doi.org/10.1111/j.13652265.2008.03288.x)

4 Dekkers OM, van der Klaauw AA, Pereira AM, Biermasz NR, Honkoop PJ, Roelfsema F, Smit JWA \& Romijn JA. Quality of life is decreased after treatment for nonfunctioning pituitary macroadenoma. Journal of Clinical Endocrinology and Metabolism 200691 3364-3369. (https://doi.org/10.1210/jc.2006-0003)

5 Andela CD, Scharloo M, Pereira AM, Kaptein AA \& Biermasz NR. Quality of life (QoL) impairments in patients with a pituitary adenoma: a systematic review of QoL studies. Pituitary 201518 752-776. (https://doi.org/10.1007/s11102-015-0636-7)

6 Kyriakakis N, Lynch J, Gilbey SG, Webb SM \& Murray RD. Impaired quality of life in patients with treated acromegaly despite long-term biochemically stable disease: results from a 5-years prospective study. Clinical Endocrinology 201786 806-815. (https://doi.org/10.1111/ cen.13331)

7 Porter ME \& Lee TH. The strategy that will fix health care. Harvard Business Review 201391 50-56, 58, 60, 150.
8 Ye VC \& Akagami R. Perioperative quality of life in Cushing's disease. Canadian Journal of Neurological Sciences 201744 69-77. (https://doi. org/10.1017/cjn.2016.295)

9 Jalessi M, Jahanbakhshi A, Amini E, Kamrava SK \& Farhadi M. Impact of nasoseptal flap elevation on sinonasal quality of life in endoscopic endonasal approach to pituitary adenomas. European Archives of OtoRhino-Laryngology 2016273 1199-1205. (https://doi.org/10.1007/ s00405-015-3729-z)

10 Little AS, Kelly DF, Milligan J, Griffiths C, Prevedello DM, Carrau RL, Rosseau G, Barkhoudarian G, Jahnke H, Chaloner C et al. Comparison of sinonasal quality of life and health status in patients undergoing microscopic and endoscopic transsphenoidal surgery for pituitary lesions: a prospective cohort study. Journal of Neurosurgery 2015123 799-807. (https://doi.org/10.3171/2014.10.JNS14921)

11 McCoul ED, Anand VK \& Schwartz TH. Improvements in site-specific quality of life 6 months after endoscopic anterior skull base surgery: a prospective study. Journal of Neurosurgery $2012 \mathbf{1 1 7} 498-506$. (https://doi.org/10.3171/2012.6.JNS111066)

12 Milian M, Honegger J, Gerlach C \& Psaras T. Health-related quality of life and psychiatric symptoms improve effectively within a short time in patients surgically treated for pituitary tumors - a longitudinal study of 106 patients. Acta Neurochirurgica 2013155 1637-1645. (https://doi.org/10.1007/s00701-013-1809-7)

13 Okamoto Y, Okamoto F, Yamada S, Honda M, Hiraoka T \& Oshika T. Vision-related quality of life after transsphenoidal surgery for pituitary adenoma. Investigative Ophthalmology and Visual Science 201051 3405-3410. (https://doi.org/10.1167/iovs.09-3763)

14 Pledger CL, Elzoghby MA, Oldfield EH, Payne SC \& Jane JA. Prospective comparison of sinonasal outcomes after microscopic sublabial or endoscopic endonasal transsphenoidal surgery for nonfunctioning pituitary adenomas. Journal of Neurosurgery $2016 \mathbf{1 2 5}$ 323-333. (https://doi.org/10.3171/2015.6.JNS142695)

15 Rioja E, Bernal-Sprekelsen M, Enriquez K, Enseñat J, Valero R, de Notaris M, Mullol J \& Alobid I. Long-term outcomes of endoscopic endonasal approach for skull base surgery: a prospective study. European Archives of Oto-Rhino-Laryngology 2016273 1809-1817. (https://doi.org/10.1007/s00405-015-3853-9)

16 Schreiber A, Bertazzoni G, Ferrari M, Rampinelli V, Verri P, Mattavelli D, Fontanella M, Nicolai P \& Doglietto F. Nasal morbidity and quality of life after endoscopic transsphenoidal surgery: a singlecenter prospective study. World Neurosurgery 2019123 e557-e565. (https://doi.org/10.1016/j.wneu.2018.11.212)

17 Tanemura E, Nagatani T, Aimi Y, Kishida Y, Takeuchi K \& Wakabayashi T. Quality of life in nonfunctioning pituitary macroadenoma patients before and after surgical treatment. Acta Neurochirurgica 2012154 1895-1902. (https://doi.org/10.1007/ s00701-012-1473-3)

18 Weigold A, Weigold IK \& Russell EJ. Examination of the equivalence of self-report survey-based paper-and-pencil and internet data collection methods. Psychological Methods 201318 53-70. (https:// doi.org/10.1037/a0031607)

19 Standaard Onderwijsindeling 2016 - Editie 2017/'18. The Netherlands: Centraal Bureau voor de Statistiek, Den Haag/Heerlen, 2016.

20 UNESCO Institute for Statistics, International Standard Classification of Education. Montreal, Canada: ISCED Fields of Education and Training 2013, 2014.

21 CBS. Zelfgerapporteerde Medische Consumptie. Gezondheid en Leefstijl, 2010.

22 Dekkers OM, Biermasz NR, Pereira AM, Roelfsema F, van Aken MO, Voormolen JHC \& Romijn JA. Mortality in patients treated for Cushing's disease is increased, compared with patients treated for nonfunctioning pituitary macroadenoma. Journal of Clinical Endocrinology and Metabolism 200792 976-981. (https://doi. org/10.1210/jc.2006-2112)

23 Dekkers OM, Hammer S, de Keizer RJW, Roelfsema F, Schutte PJ, Smit JWA, Romijn JA \& Pereira AM. The natural course of 
non-functioning pituitary macroadenomas. European Journal of Endocrinology 2007156 217-224. (https://doi.org/10.1530/ eje.1.02334)

24 Biermasz NR, Dekkers OM, Voormolen J, de Keizer RJW, Neelis KJ, Wiggers-de Bruine FT, Smit JW, Pereira AM \& Romijn JA.

Transsphenoidal resection of pituitary adenomas: long-term results from the Leiden University Medical Center. Nederlands Tijdschrift voor Geneeskunde $2008 \mathbf{1 5 2} 2565-2570$.

25 Norman GR, Sloan JA \& Wyrwich KW. Interpretation of changes in health-related quality of life: the remarkable universality of half a standard deviation. Medical Care 200341 582-592. (https://doi. org/10.1097/01.MLR.0000062554.74615.4C)

26 Andela CD, Scharloo M, Ramondt S, Tiemensma J, Husson O, Llahana S, Pereira AM, Kaptein AA, Kamminga NGA \& Biermasz NR. The development and validation of the Leiden Bother and Needs Questionnaire for patients with pituitary disease: the LBNQpituitary. Pituitary 201619 293-302. (https://doi.org/10.1007/ s11102-016-0707-4)

27 Ware JE \& Sherbourne CD.The MOS 36-item short-form health survey (SF-36). Medical Care 199230 473-483.

28 EuroQoL Group. EuroQol - new facility for the measurement of health-related quality of life. Health Policy 199016 199-208. (https:// doi.org/10.1016/0168-8510(90)90421-9)

29 M Versteegh M, M Vermeulen K, M A A Evers S, de Wit GA, Prenger R \& A Stolk E. Dutch tariff for the five-level version of EQ-5D. Value in Health 201619 343-352. (https://doi.org/10.1016/j. jval.2016.01.003)

30 Mangione CM, Lee PP, Gutierrez PR, Spritzer K, Berry S, Hays RD $\&$ National Eye Institute Visual Function Questionnaire Field Test Investigators. Development of the 25-list-item National Eye Institute Visual Function Questionnaire. Archives of Ophthalmology 2001119 1050-1058. (https://doi.org/10.1001/archopht.119.7.1050)
31 van Roijen L, Essink-Bot ML, Koopmanschap MA, Bonsel G \& Rutten FF. Labor and health status in economic evaluation of health care. The Health and Labor Questionnaire. International Journal of Technology Assessment in Health Care 199612 405-415. (https://doi. org/10.1017/S0266462300009764)

32 Little AS, Jahnke H, Nakaji P, Milligan J, Chapple K \& White WL. The anterior skull base nasal inventory (ASK nasal inventory): a clinical tool for evaluating rhinological outcomes after endonasal surgery for pituitary and cranial base lesions. Pituitary 201215 513-517. (https://doi.org/10.1007/s11102-011-0358-4)

33 Piccirillo JF, Merritt MG \& Richards ML. Psychometric and clinimetric validity of the 20-item sino-nasal outcome test (SNOT20). Otolaryngology: Head and Neck Surgery 2002126 41-47. (https:// doi.org/10.1067/mhn.2002.121022)

34 Eekhout I, de Vet HC, de Boer MR, Twisk JW \& Heymans MW. Passive imputation and parcel summaries are both valid to handle missing items in studies with many multi-item scales. Statistical Methods in Medical Research 201827 1128-1140. (https://doi. org/10.1177/0962280216654511)

35 Lindsay JR, Nansel T, Baid S, Gumowski J \& Nieman LK. Longterm impaired quality of life in Cushing's syndrome despite initial improvement after surgical remission. Journal of Clinical Endocrinology and Metabolism 200691 447-453. (https://doi.org/10.1210/jc.2005-1058)

36 Prinsen CAC, Vohra S, Rose MR, Boers M, Tugwell P, Clarke M, Williamson PR \& Terwee CB. How to select outcome measurement instruments for outcomes included in a 'Core Outcome Set' - a practical guideline. Trials 201617 449. (https://doi.org/10.1186/ s13063-016-1555-2)

37 Webb SM, Santos A, Resmini E, Martínez-Momblán MA, Martel L \& Valassi E. Quality of life in Cushing's disease: a long term issue? Annales d'Endocrinologie 201879 132-137. (https://doi.org/10.1016/j. ando.2018.03.007)

Received 8 May 2019

Revised version received 18 July 2019

Accepted 23 July 2019 